\title{
The Apsidal Motion Test in Eclipsing Binaries
}

\section{Alvaro Giménez}

Research and Scientific Support Department, ESA, ESTEC, Noordwijk, the Netherlands

\begin{abstract}
The use of eccentric eclipsing binaries to test stellar internal structure models, as well as the equations of motion provided by General Relativity, is reviewed. Close to 80 years have elapsed since the first ideas were produced in this field and many results have been obtained since then. It appears that, in general, a good understanding of stellar structure within the main sequence is available while the same level of knowledge can not be claimed beyond the termination age. The equations of general relativity could not be disproved with observational data though some systems cannot still be fully explained. In the near future, the analysis of evolved systems, very low mass stars, the effects of tidal resonances and the presence of third bodies has to be further explored. In addition, the analysis of large data bases obtained by means of extensive photometric surveys will certainly change the picture from an observational point of view.
\end{abstract}

Keywords. Eclipsing binaries, Eccentric Binaries, Apsidal Motion, Internal Structure, General Relativity

\section{Introduction}

The purpose of this review is to present briefly the history of the apsidal motion test in the field of close binaries as well as the current status of the subject, latest results and prospects for the future. The initial idea to measure the motion of the periastron of binary stars in order to have some insight into their internal structure was given by Russell (1928). His problem was the accumulation of observational data on eclipsing binaries with spectroscopic orbits leading to values of the average density of the stars well below that needed to achieve configurations in hydrostatic equilibrium. It was clear that the central density concentration had to be much higher than the average value. In eccentric binaries, the behavior of the orbit is ruled by the distortion of the components and this is a function of the internal density concentration as well as the mass ratio and the separation of the components. Stellar distortion, or deviation from the point-like behavior, is responsible for the secular movement of the periastron and its observational measurement should obviously lead to an empirical measurement of the level of the density concentration.

The idea was right but the proposed equations for the apsidal motion were not fully developed. A debate was initiated about the proper treatment of the problem and Kopal (1936) proposed to measure the stellar distortion directly by means of the photometric variations outside of eclipse, but the existence of additional effects, like reflection, precluded to reach reliable conclusions. On the other hand, stellar models were not available to predict the rate of motion of the periastron though Chandrasekhar (1933) contributed to solve this by computing the internal density concentration, and corresponding distortions, for polytropic models. The equations of motion were revised by Cowling (1938) who identified the internal structure parameter $k_{2}$ to be related to the apsidal motion rate through the fifth power of the relative radii of the stars. Accurate absolute 
dimensions were therefore necessary for any reliable interpretation of the observational data. Kopal (1938) contributed to the development of the equations by introducing the effects of libration and Sterne (1939a) extended the work by Cowling (1938) using terms up to $r^{9}$ while producing the necessary tools to analyze the observations of eclipse timings under the assumption that the line of sight remains within the orbital plane.

Sterne (1939b) could thus deliver the first comparison of polytropic models with observations, collected for 5 binary systems. The best agreement was found for a polytropic index $n=3$, also known as the standard model. Sterne was nevertheless well aware that the comparison of non-accurate data with unrealistic models could not lead to reliable conclusions and the method did not advance further. In parallel, Levi-Civita (1937) had found the equations for the relativistic motion of eccentric binary stars to be equivalent to those corresponding to the perihelion motion of the planet Mercury. The results were probably academic at the time but proved to be applicable years later as described in Section 3 .

Schwarzschild (1958) revitalized the apsidal motion test by making a comparison using reliable observational data for 8 systems and new internal structure models based on realistic physical assumptions. The result then was that the observations were indicative of much more centrally condensed stars than actually predicted. Schwarzschild already noticed that the situation improved, but was far from solved, taking into account stellar evolution. New detailed equations for the apsidal motion were given by Kopal (1959) including their modification when non-synchronized rotation, inclined axes, or nodal variations are taken into account. Kopal (1965) also revised the situation of the comparison between models and observations increasing the number of binary systems to 14 but being only able confirm the disagreement, even considering cases just within the main sequence.

In the following decade, the apsidal motion test was revisited by several authors. It is interesting to note the critical review by Batten (1973) and the evolutionary considerations by Petty (1973). Stothers (1974) introduced in the discussion the effects of the adopted opacities and stellar rotation, from the point of view of the models computations, both in fact leading to more centrally concentrated stellar mass distribution, or lower values of the internal structure constants $k_{2}$, as actually observed. On the other hand, O'Dell (1974) questioned the predicted size of the cores of massive stars. Finally, Kopal (1978) reviewed the dynamical behavior of close binaries with no definitive solution to the disagreement between models and observations.

The interest for further analysis of eccentric eclipsing binaries showing apsidal motion increased considerably as a consequence of all these studies. Giménez (1981a) tried to put together all the accumulated data and made a critical discussion of the comparison with the best available theoretical models. The new approach included a revision of the selection of the data sample. Only well-detached systems with well-determined eccentricities and clean light curves were used. From an observational point of view, the determination of apsidal motion rates was purely based on the measurement of the changing relative position of deep eclipses. Further, in order to make the comparison with the relevant models, accurate absolute dimensions for the component stars were used. Even with these severe constraints, the number of systems used in the comparison could be increased to 20 as discussed in detail by Giménez (1981b).

The results, also shown by Giménez \& Garcia-Pelayo (1982), clearly indicated the strong dependence of the variation of $k_{2}$ with age and, to a lower degree, with the adopted opacities. It was then clear that new modern evolutionary models were needed. For the first time, the observational data were precise enough to identify the source of the problem in the apsidal motion test. 


\section{The quadrupole term}

During the eighties the effort was concentrated in the computation of new models. The old opacities by Cox \& Stewart (1970), adopted by Hejlesen (1980), were very useful for the comparison with absolute dimensions obtained from well-detached eclipsing binaries (see Andersen 1991), though they presented a problem with the fit of the effective temperatures for low mass stars and the internal structure constants were only published years later (Hejlesen 1987). The solution to this problem came with the use of better opacity tables. Jeffery (1984) computed models using those by Carson (1976) that predicted more centrally concentrated stars, but proved later to be based on unrealistic assumptions. More detailed computations of the internal structure constants were published by Claret \& Giménez (1989) but the real improvement was made when the new Los Alamos opacities by Rogers \& Iglesias (1992) were available and the corresponding computations were made by Claret \& Giménez (1992), taking also into account the effect of a moderate amount of convective core overshooting.

In parallel, the equations for the determination of apsidal motion rates by means of the analysis of the position of the eclipses were revised by Giménez \& Garcia-Pelayo (1983) and new cases of double-lined eclipsing binaries with eccentric orbit could be added to the data sample available for the comparison with the models. During the same period of time, several studies were carried out to assess tidal effects in the rotation of the component stars. This parameter was known to play an important role in the determination of the internal structure constants as well as in their modelling.

Using 24 binary systems and the latest models, a detailed comparison of the $k_{2}$ internal structure constants was published by Claret \& Giménez (1993). The adopted procedure first verified that the observed effective temperatures of the component stars were in good agreement with those predicted by the models and these, in addition, provided the same evolutionary age for the primary and the secondary component of each system. Finally, the observed rotational velocities were compared with the orbital period. As a result the quality of the data, and the goodness of the models to reproduce them, could be assessed.

The comparison of the internal structure constants showed a much better agreement than in previous studies, certainly as a result of the consideration of stellar evolution. Nevertheless, a systematic difference could still be seen, again in the sense of stars being more centrally concentrated than predicted. The difference was found also to be a function of evolution but with a much smaller amplitude than that indicated by Giménez \& GarciaPelayo (1982). In any case, the disagreement was reduced to just three cases with the lowest data quality (which were also the more evolved ones as indicated by Giménez 1984) and the main part of the data sample was very well reproduced, with no systematic effect when the models were corrected for the effect of rotation. It has already been mentioned that rotation has to be taken into account since models, generally computed for nonrotating configurations, are compared to real, rotating, stars in the apsidal motion test. Claret (1999) has analyzed the issue in detail, computing rotating models, and provided a good confirmation of the correction term given by Stothers (1974). Moreover, the situation with the more evolved systems could be improved by assuming some degree of convective core overshooting, fully consistent with independent tests of the width of the main sequence by Andersen, Nordström \& Clausen (1990).

Further studies were obviously required and were developed in three different areas. New lists of candidates to be monitored superseded those by Kopal (1978) and Giménez \& Delgado (1980), and were given by Hegedus (1988), Giménez (1994), Petrova \& Orlov (1999) and Hegedus, Giménez \& Claret (2005). They have allowed the systematic 
monitoring of more than 100 eclipsing binaries in order to determine accurate apsidal motion rates and absolute dimensions. The second area of development was an improvement in the methods used to analyze the data. Lacy (1992) proposed an exact iterative method while Giménez \& Bastero (1995) extended the earlier equations by Giménez \& Garcia-Pelayo (1983), and van Hamme \& Wilson (1998) added new ideas using the simultaneous solution of all the observational evidence rather than just the position of the eclipses. As a third area of research, new detailed theoretical models were computed in the 1995-2005 decade for a wide range of masses and chemical compositions by Claret (see e.g. Claret 1995), allowing the comparison with observational data for different degrees of metallicity, rotation, and convective overshooting.

The latest general comparison of models with observational data has been published by Claret \& Willems (2003). Allowing the fit of models for the best chemical composition combination led to an excellent comparison of the predicted and observed effective temperatures as well as the evolutionary ages of the two components of each system. The comparison of the internal structure constants showed no significant difference at all when a moderate degree of convective core overshooting was assumed and thus the long fight to reconcile models and observations could be closed. A reasonable agreement was definitely found provided that a) the initial chemical composition is taken into account, b) the empirical values of the internal structure are corrected for rotational effects, and c) some amount of convective core overshooting is considered (see also Giménez et al. 1999). The determination of the initial chemical composition for a sample of binary stars allowed in addition the estimation of the fundamental chemical enrichment law in our Galaxy. This had already been shown by Ribas et al. (2000a) for a wider sample of binary systems and showed an excellent agreement with results obtained through other approaches. A similar use of the test for the required degree of convective core overshooting allowed Ribas, Jordi \& Giménez (2000) to show a first attempt to look into its variation with mass.

Two possible complications were nevertheless identified that could bias the apsidal motion test assumptions and give unexpected results in some individual cases. The presence of an unseen third body in a given system could affect the observational data and the assumption of equilibrium tides made by Cowling and Sterne could not be valid. In fact, the importance of dynamical and resonance tides was already pointed out by Papaloizou \& Pringle (1980) and discussed by Quataert, Kumar \& Ao (1996), Smeyers, Willems \& van Hoolst (1998), and Smeyers \& Willems (2001). Nevertheless, when the modified equations are applied to eccentric binaries, like those currently used in the apsidal motion test, Claret \& Willems (2003) showed that no significant effect should be expected and the classical approach can be safely used within the level of the observational uncertainties. On the other hand, the discovery of third bodies in the adopted sample of eccentric binaries, mostly young systems, increased considerably with the precision of the observational data. Light-time effect was mainly used together with the need for third-light in the light curve, to detect the presence of these companions. The equations by Martynov (1948) could be applied to correct the observed apsidal motion rates. Nevertheless, the existence of unseen bodies remains a source of uncertainty in the test. A classical example is AS Cam, an anomalous case that could be explained with a non-coplanar third body, as discussed by Khodykin \& Vedenesev (1994) and Kozyreva et al. (1999).

\section{The relativistic term}

Studies looking for the internal density concentration of the stars corrected the observed apsidal motion rates for the contribution due to the relativistic equations of 
motion, as provided in early times by Levi-Civita (1937). But the test of general relativity is an interesting subject on its own and apsidal motion measurements can also be used for this purpose. Rudkjobing (1959) already suggested that the massive eccentric binary DI Her, with an orbital period of around 10 days, could be an excellent observational target. Unfortunately, despite many attempts, the detection of the relativistic term in the apsidal motion of DI Her failed to give the expected results. In 1982, Giménez \& Scaltriti (1982) could measure reliably the apsidal motion of the system V889 Aql to be in good agreement with the equations of General Relativity. However, the system was less massive than the original target suggested by Rudkjobing (1959) or those mentioned by Koch (1973). Koch indeed proposed 5 candidates, 2 of them with good absolute dimensions, but none with a clear determination of the apsidal motion rate. Giménez (1985) subsequently showed that the key parameter is not the total mass of the system but the relative radii of the component stars since the newtonian apsidal motion rate decreases much faster than the relativistic term with this parameter. Following this argument, a new list of 23 candidate systems for the relativistic test was put together by Giménez (1985) leading necessarily to very slow rotation periods and thus difficulties in their measurement, as discussed by Claret (1997). By 1985, already 5 systems had been studied with a relativistic term dominating the total apsidal motion rate: 4 in good agreement with General Relativity (V889 Aql, EK Cep, V1143 Cyg, and VV Pyx) and one in clear disagreement (DI Her), as found by Guinan \& Maloney (1985). A new approach to formalize gravitation by Moffat (1989) was then used to explain the problem though, when applied to all systems with apsidal motion, the use of the same parametrization failed to fit the otherwise good agreement in the predicted and observed internal structure constants as shown by Claret (1997).

Dynamical tides are less important in the well-detached systems selected for the relativistic test than in closer binaries so that they could not be used to explain the disagreement of DI Her. Non-synchronization of the rotational axis with that perpendicular to the orbit was proposed by Shakura (1985) but only the presence of a third body in an almost orthogonal orbit with respect to the orbital plane of the eclipsing system could lead to the observed apsidal motion rate, as indicated by Khodykin \& Vedenesev (1994) in the case of AS Cam, and discussed by Hsuan \& Mardling (2006) for DI Her. The effect of a third body is an important issue in the study of apsidal motions and several systems in the list of Giménez (1985) have been later found to have companions. The expected effect is nevertheless to increase the apsidal motion rate rather than to slow it, as indicated by the equations of Martynov (1948) in the case of coplanar orbits. A simple guess indicates that at least $1 / 3$ of all binaries in the referred list of candidates may have a third body, and possibly $1 / 2$.

A revision of the situation has been carried out with inputs from the literature collected in the last two decades. The list of candidate systems now include 36 binaries, instead of 23, with 25 of them having good absolute dimensions, instead of 9. Realistic apsidal motion determinations are available for 16 binaries in the sample, compared to the previous 5 and a new comparison with theoretical predictions could be thus attempted. Preliminary results show that a good agreement is found for 12 out of the mentioned 16 cases and they are shown in Figure 1.

Giménez (1985) had found that 4 systems were in agreement with the theory and 1 in disagreement. The situation now is that 12 systems are in agreement and 4 failed the comparison (DI Her, BW Aqr, SW CMa and ES Lac). Although the statistics of failures remains more or less the same, the interesting point is that all except DI Her present a faster than expected apsidal motion; i.e., they can be easily explained with a, not yet seen, coplanar third body. The case of DI Her remains the only odd case requiring a third 


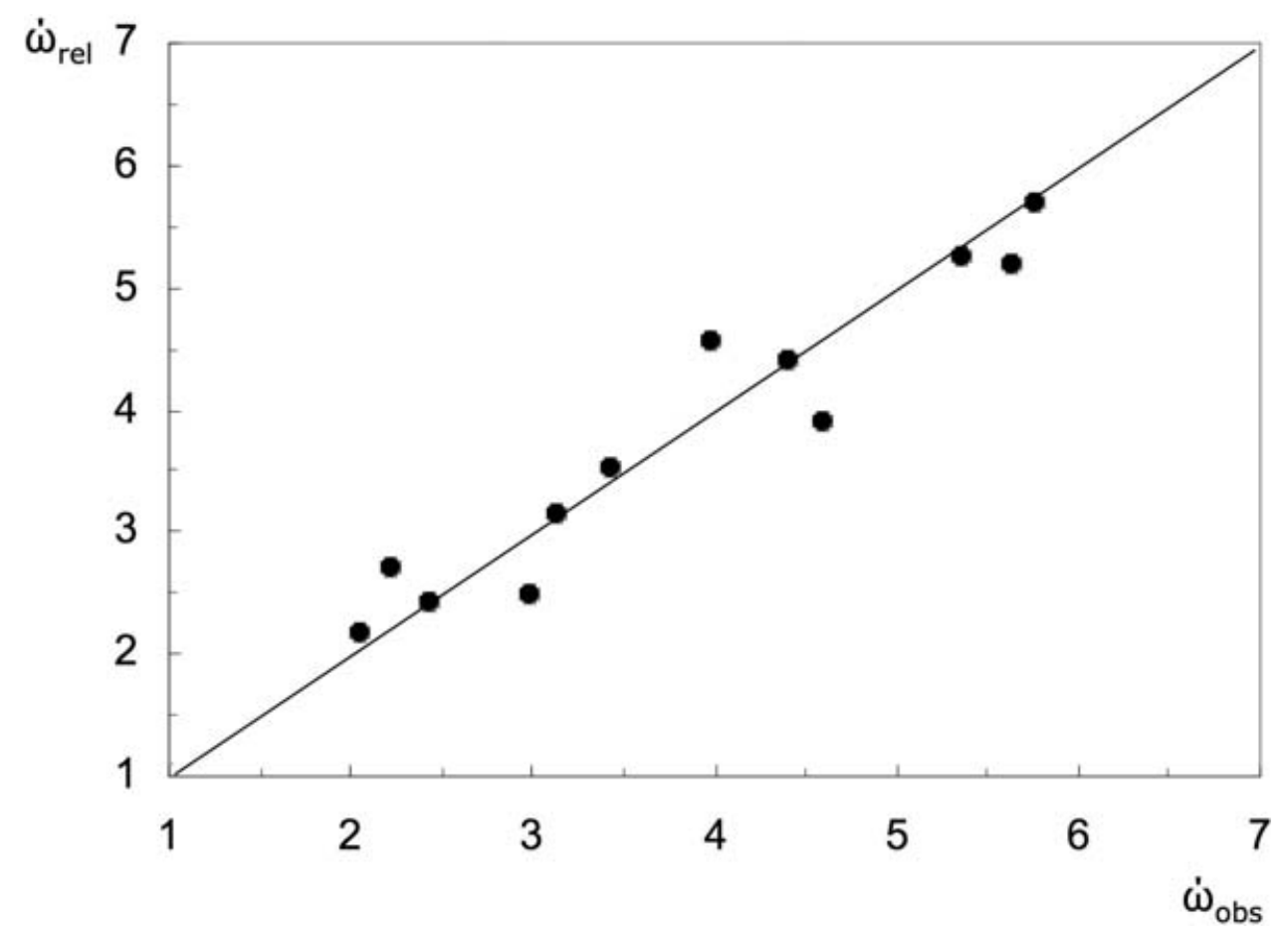

Figure 1. Comparison of the predicted and observed relativistic term in the apsidal motion rate of selected eclipsing binaries.

body with an orbit perpendicular to that of the eclipsing system. One case is of course not enough to disprove a solid theory and, in addition, other cases are known to have non coplanar third bodies, like that of SS Lac that stopped showing eclipses due to the induced change in the inclination of the orbit (Torres 2001) or the previously mentioned AS Cam.

\section{The future}

The field of apsidal motion studies in eclipsing binaries is not over despite the advance of the last years. The first point requiring further analysis is linked to the fact that most of the systems studied up to now cover medium mass stars within the main sequence and close to solar chemical composition. An extension of the test has therefore to be carried out in other domains of the HR diagram. The study of very low mass stars, like CM Dra, OGLE-TR-122 (Pont et al. 2005) or the recently discovered brown-dwarf binary (Stassun, Mathieu \& Valenti 2006), will certainly help understanding the structure of purely, or almost so, convective configurations. On the other side, the analysis of evolved massive systems, like V380 Cyg or V453 Cyg, recently studied by Southworth, Maxted \& Smalley (2004), will provide a better knowledge of convective core overshooting, including its variation with mass and age. Finally, binaries formed in a different environment, or metallicity, like HV 2274 in the LMC studied by Ribas et al. (2000b), will provide an insight into the internal structure of extragalactic stars.

In these studies it is clear that increased efforts have to be devoted to the determination of accurate absolute dimensions for all systems showing apsidal motion and, if possible, for all eccentric eclipsing binaries, as potential candidates for the test of the models. 
Of course, new minima should be obtained for the most interesting cases, in order to determine accurate apsidal motion periods. One of the most important aspects in the coming years for the comparison between observations and stellar structure models will be to assess the effect of third bodies in the systems studied. To look carefully for any indication of a third body, either directly, through their effect in the light curve or in the variation of the eclipse timings, will be critical.

The observational constraints imposed by Giménez (1981a) for the apsidal motion test, can now be partly lifted thanks to the much better observational data available. An example is the clean light curve of the eccentric system RZ Eri shown by Burki, Kviz \& North (1992) with heavily spotted components. But the future of the test will be mainly driven by the large increase in the discovery of new eclipsing binaries. The first way to go is certainly the systematic and in depth exploitation of existing data bases of light curves of eclipsing binaries. The use of the Hipparcos data has shown, despite its low photometric precision, how the determination of additional eclipse timings is critical in the determination of accurate apsidal motion rates (Otero 2003). Large surveys in crowded fields, looking for microlensing events, like OGLE, MACHO and EROS, have discovered thousands of new eclipsing binaries, many of them showing eccentric orbits and thus providing many more candidate systems than those in classical catalogues. Identifying those that best suit the kind of fundamental tests mentioned above will be the main task in the coming years. A good example of the determination of apsidal motion periods from binaries discovered in a survey of the LMC, using automated algorithms, has been shown by Michalska \& Pigulski (2006). The newly discovered eccentric eclipsing binaries in the Magellanic Clouds and beyond, like the system in M33 studied by Bonanos et al. (2006), will open further possibilities for testing stellar structure outside our own Galaxy. But another area of interest that I would like to emphasize is the study of dynamical tides and resonant oscillations in eccentric systems known to have pulsating components. The precision and amount of data accumulated for this type of eclipsing systems, neglected for some time, has been increasing recently and the apsidal motion test can provide very important new pieces of information.

\section{References}

Andersen, J. 1991, Astron. \& Astrophys. Reviews, 3, 91

Andersen, J., Nordström, B., \& Clausen, J.V. 1990, ApJ, 363, L33

Batten, A.H. 1973, Binary and Multiple Systems of Stars, Pergamon Press, New York

Bonanos, A.Z. et al. 2006, , preprint in astro-ph/0606279

Burki, G., Kviz, Z., \& North, P. 1992 A\&A, 256, 463

Carson, T.R. 1976, Ann. Rev. Astron. Astrophys., 14, 95

Chandrasekhar, S. 1933, MNRAS, 93, 449

Claret, A. 1995, A\&ऽA Suppl., 109, 441

Claret, A. 1997, $A \mathscr{E} A, 327,11$

Claret, A. 1999, A\&SA, 350, 56

Claret, A. \& Giménez, A. 1989, A\&A, 81, 1

Claret, A. \& Giménez, A. 1992, A\&SA Suppl., 96, 255

Claret, A. \& Giménez, A. 1993, A\&A, 277, 487

Claret, A. \& Willems, B. 2003, AESA, 410, 289

Cowling, T.G. $1938 M N R A S, 98,734$

Cox, A.N. \& Stewart, J.N 1970, ApJ Suppl., 19, 243

Giménez, A. 1981a, in Photometric and Spectroscopic Binary Systems, NATO Advanced Institute Series, Eds. E.B. Carling \& Z. Kopal, Kluwer, Dordrecht, 511

Giménez, A. 1981b, PhD Thesis, University of Granada 
Giménez, A. 1984, Observational Tests of the Stellar Evolution Theory, IAU Symp. 105, Eds. A, Maeder \& A. Renzini, Reidel, Dordrecht, 419

Giménez, A. 1985, ApJ, 297, 405

Giménez, A. 1994, Exp. Astron., 5, 91

Giménez, A. \& Bastero, M. 1995, Astrophys. Space Sci., 226, 99

Giménez, A. \& Delgado, A. 1980, I.B.V.S., IAU Comm. 27, No. 1815

Giménez, A. \& Garcia-Pelayo, J.M. 1982, Binary and Multiple Stars as Tracers of Stellar Evolution, Eds. Z. Kopal \& J. Rahe, Reidel, Dordrecht, 37

Giménez, A. \& Garcia-Pelayo, J.M. 1983, Astrophys. Space Science, 92, 203

Giménez, A. \& Scaltriti, F. 1982, A\&Ap, 115, 321

Giménez, A., Claret, A., Ribas, I., \& Jordi, C. 1999, Theory and Tests of Convection in Stellar Structure, Eds. A. Giménez, E.F. Guinan \& B. Montesinos. ASP Conf. Series, 173, 41

Guinan, E.F. \& Maloney, F.P. 1985, AJ, 90, 1519

van Hamme, W.V. \& Wilson, R.E. 1998, BAAS, 30, 1402

Hegedus, T. 1988, Bull. Inf. CDS, 35, 15

Hegedus, T., Giménez, A., \& Claret, A. 1985, Tidal Evolution and Oscillations in Binary Stars, Eds. A. Claret, A. Giménez \& J.P. Zahn, ASP Conf. Series, Vol. 333, 88

Hejlesen, H.E. 1980, A\&̈A Suppl., 39, 347

Hejlesen, H.E. 1987, A\&\&A Suppl., 69, 251

Hsuan, K. \& Mardling, R.A. 2006, Astrophys. Space Sci., in press

Jeffery, S.F. 1984 MNRAS, 207, 323

Khodykin, S.A. \& Vedenesev, V.G. 1994, ApJ, 475, 798

Koch, R.H. 1973, ApJ, 183, 275

Kopal, Z. 1936, MNRAS, 96, 854

Kopal, Z. 1938, MNRAS, 98, 448

Kopal, Z. 1959, Close Binary Systems, Chapman \& Hall, London

Kopal, Z. 1965, Advances in Astron. ES Astrophys., 3, 89

Kopal, Z. 1978, Dynamics of Close Binary Systems, Reidel Publ., Dordrecht

Kozyreva, V.S., Zakharov, A.I., \& Khaliullin, Kh.F. 1999, IBVS, No. 4690

Lacy, C. 1992, AJ, 104, 2213

Levi-Civita, T. 1937, Amer. J. Mathem., 59, 225

Martynov, D. Ya. 1948, Izv. Engelhardt Obs. Kazan, No. 25

Michalska, G. \& Pigulski, A. 2006, A\& $A$, preprint in astro-ph/0501380

Moffat 1989, Phys. Rev. D, 39, 474

O'Dell, A.P. 1974, ApJ, 192, 417

Otero 2003, IBVS, 5482

Papaloizou, J. \& Pringle, J.E. 1980, MNRAS, 193, 603

Petrova, A.V. \& Orlov, V.V. 1999, AJ, 117, 587

Petty, A.F. 1973, Astrophys. Space Sci., 21, 189

Pont, F., Melo, C.H.F., Bouchy, F., Udry, S., Queloz, D., Mayor, M., \& Santos, N.C. 2005, A\&A, 433, L21

Quataert, E.J., Kumar, P., \& Ao, C.O. 1993, ApJ, 463, 284

Ribas, I., Jordi, C., \& Giménez, A. 2000, MNRAS, 318, L55

Ribas, I., Jordi, C., Torra, J., \& Giménez, A. 2000a, MNRAS, 313, 99

Ribas, I., Guinan, E.F., Fitzpatrick, E.L., de Warf, L.E., Maloney, F.P., Maurone, P.A., Bradstreet, E.H., Giménez, A., \& Pritchard, J.D. 2000b, ApJ, 528, 692

Rogers, F.J. \& Iglesias, C.A. 1992, ApJ Suppl., 79, 507

Rudkjobing, M. 1959, Ann. d'Astroph., 22, 111

Russell, H.N. 1928, MNRAS, 88, 642

Schwarzschild, M. 1958, Structure and Evolution of the Stars, Princeton University Press

Shakura, N.I. 1985 Sov. Astr. Lett., 11, 225

Smeyers, P., Willems, B., \& van Hoolst, T. 1998, A\&A, 335, 622

Smeyers, P. \& Willems, B. 2001, A\&A, 373, 173

Southworth, J., Maxted, P.F.L., \& Smalley, B. 2004, MNRAS, 351, 1277

Stassun, K.G., Mathieu, R.D., \& Valenti, J.A. 2006, Nature, 440, 311 
Sterne, T.E. 1939a, MNRAS, 99, 451

Sterne, T.E. 1939b, MNRAS, 99, 662

Stothers, R. 1974, ApJ, 194, 651

Torres, G. 2001, AJ, 121, 2227

\section{Discussion}

Guillem AngladA-Escudé: What is the timing accuracy required for your studies? Does it really depend very strongly on color information?

GiménEz: It depends, of course, on the period of each particular system. The photometry and the deepness of the light curve and the eclipses ultimately determine your timing precision. Multicolor minima are used to accurately calibrate minima's asymmetries that can potentially perturb the instant of minima estimation.

NANCY EvANS: Just to let you know about a little more information we will provide to let you interpret your very interesting results. We (with Guinan, Ribas, Fitzpatrick, etc.) have got the FUSE spectra of some of the hottest systems to study the temperatures.

GiménEZ: This is excellent news.

Robert Wilson: Could differential rotation be a significant factor? Tidal locking makes the outer envelope rotate at the orbital rate, but stay at another rate in the somewhat deeper interior.

GimÉnEZ: Indeed some observational results of the apsidal motion test can be interpreted as an effect of convective core overshooting but could also be understood by any physical process that produces more massive cores. Differential rotation, if it is faster inside the star, may provide one of these processes. 